\title{
Ordering and shape of self-assembled InAs quantum dots on GaAs(001)
}

\author{
K. Zhang, ${ }^{\text {a) }}$ Ch. Heyn, and W. Hansen \\ Institut für Angewandte Physik, Universität Hamburg, Jungiusstraße 11, D-20355 Hamburg, Germany \\ Th. Schmidt ${ }^{\text {b) }}$ and J. Falta ${ }^{\text {b) }}$ \\ Hamburger Synchrotronstrahlungslabor HASYLAB am Deutschen Elektronen-Synchrotron DESY, \\ Notkestraße 85, D-22603 Hamburg, Germany
}

(Received 8 December 1999; accepted for publication 22 February 2000)

\begin{abstract}
Quantitative grazing-incidence small-angle x-ray scattering experiments have been performed on self-assembled InAs quantum dots (QDs) grown by molecular-beam epitaxy. We find pronounced nonspecular diffuse scattering satellite peaks with high diffraction orders, indicating a lateral ordering in the spatial positions of the InAs QDs. The mean-dot-dot distance and correlation lengths of the dot lateral distribution are found to be anisotropic. We observe the sharpest dot distribution in the [110] direction. Additional broad diffraction peaks are observed and associated with dot facet crystal truncation rods of the $\{111\}$ and $\{101\}$ facet families. This suggests an octagonal-based dot shape. (C) 2000 American Institute of Physics. [S0003-6951(00)02716-9]
\end{abstract}

In semiconductor systems, artificially designed confinement potentials are employed to study and control optoelectronic properties. ${ }^{1}$ Presently, the smallest and strongest confinement in three dimensions is achieved in so-called selfassembled systems. Self-assembling mechanisms occur, e.g., in the heteroepitaxy of materials with a large lattice mismatch. In the Stranski-Krastanov growth mode, e.g., of InAs on GaAs substrates, small InAs islands are formed if a critical layer thickness of an InAs wetting layer is exceeded. The process is thought to be entirely driven by the strain relaxation induced by the lattice mismatch of up to $7 \%$. Studies with atomic force microscopy (AFM) or transmission electron microscopy (TEM) reveal that at low InAs coverage the quantum dots (QDs) are coherently strained, dislocation-free, and have diameters of a few $10 \mathrm{~nm}$ with a surprisingly narrow size distribution. ${ }^{2}$ This allows intriguing studies of atomic-like electron states within the InAs QDs. Furthermore, there is a great effort to characterize, understand, and improve the structural properties of such QD nanostructures, as reported recently. ${ }^{3-7}$ Experimentally, high-resolution $\mathrm{x}$-ray diffraction plays an important role in the study of the structure in the vertical stacking InAs QD samples. ${ }^{3}$ However, as to a single-dot-layer structure, there is little experimental knowledge about the ordering character of QDs, since the total amount of volume fraction of InAs dots is so small that, generally, the scattering intensity from InAs QDs is very weak.

Also, the shape of InAs QD is debated intensely, since an accurate calculation of the electronic structure depends critically on the exact QD shape. Square-based pyramid ${ }^{8}$ lens shapes and conical dot shapes ${ }^{9}$ have been proposed and complex numerical models have been developed to predict the dot shape, e.g., Ref. 10, however, these investigations so far did not provide a definite picture of the QD shape.

In order to measure the ordering and shape of an un-

\footnotetext{
${ }^{a}$ Electronic mail: zhang@physnet.uni-hamburg.de

${ }^{b)}$ Present address: Institut für Festkörperphysik, Universität Bremen, Postfach 330440, D-28334 Bremen, Germany.
}

capped InAs QD single layer, we have performed highresolution grazing-incidence small-angle $\mathrm{x}$-ray scattering (GISAXS) experiments ${ }^{11}$ with high-flux synchrotron radiation. The grazing-incidence technique probes the crystal surface up to depths of only a few nanometers so that the volume fraction of InAs probed is much larger comparing to conventional x-ray techniques. Furthermore, as compared to AFM measurements, a relatively large area contributes to coherent scattering so that the distribution can be determined with improved statistics.

Our samples have been prepared by molecular-beam epitaxy. A 200-nm-thick GaAs buffer layer was deposited on a $\mathrm{GaAs}(001)$ substrate at $600^{\circ} \mathrm{C}$, and then the substrate temperature was decreased to $500^{\circ} \mathrm{C}$ for InAs growth. The amount of InAs deposited corresponds to a coverage of 2.1 ML. The In flux was calibrated to be $0.1 \mathrm{ML} / \mathrm{s}$ with reflection high-energy electron diffraction (RHEED) oscillations. In order to improve the homogeneity of the InAs growth, the GaAs substrate was rotated during layer deposition. As soon as the growth was finished, the sample was cooled down to room temperature immediately. The misorientation of the wafer surface with respect to the (001) crystal plane is determined to be about $0.01^{\circ}$ by x-ray topographic technique.

The topology of InAs QDs is investigated by AFM with a cantilever tip of $10 \mathrm{~nm}$ diam. Figure 1 shows a zoom into a $1 \times 1 \mu \mathrm{m}^{2}$ AFM image. The AFM measurements indicate: (i) the dots exhibit a narrow size distribution and a nearly homogeneous position distribution; (ii) the dot density is about $3.8 \times 10^{10} \mathrm{~cm}^{-2}$; (iii) the average dot height and lateral size are about $4 \pm 1$ and $30 \pm 10 \mathrm{~nm}$, respectively; and (iv) by Fourier transformation of the $1 \times 1 \mu \mathrm{m}^{2}$ image, the meandot-dot distance in the [110] direction is estimated to be about $67 \pm 8 \mathrm{~nm}$, while in the [1 10$]$ direction the mean-dotdot distance can hardly be resolved due to the absence of a pronounced maximum in the power spectrum.

GISAXS measurements were performed at the Hamburg Synchrotron Radiation Laboratory (HASYLAB) undulator beamline BW1 at a wavelength of $1.17 \AA$. The incidence angle to the sample surface was chosen to $0.18^{\circ}$, which is 


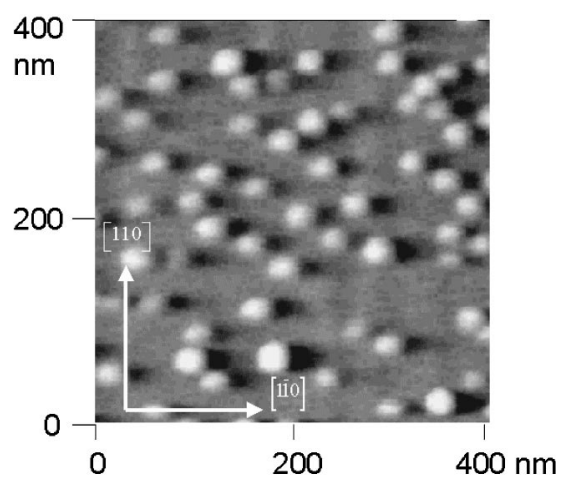

FIG. 1. Typical AFM image of a self-assembled InAs QD with 2.1 ML InAs coverage. Left shading at an angle of $45^{\circ}$ to the horizontal direction is processed to the AFM image for clarity.

just below the total external reflection angle of $0.20^{\circ}$. Intensity profiles were collected by a position-sensitive detector (PSD) mounted parallel to the sample surface. A PSD resolution of $2.1 \times 10^{-3} \AA^{-1}$ is achieved in the direction parallel to the sample surface. With this resolution, mean-dot-dot distances of up to $300 \mathrm{~nm}$ can be determined.

To characterize the lateral distribution of self-assembled InAs QDs, GISAXS experiments were performed for different sample azimuthal orientations $\Omega$ in an angle range of $-10^{\circ}$ to $110^{\circ}$ in steps of $5^{\circ}$. The angle $\Omega=0^{\circ}$ when the [110] direction is oriented parallel to the incoming beam. As the PSD is perpendicular to the incoming beam, it probes scattering vectors $\mathbf{q}_{\|}$(and thus structural properties, e.g., at $\Omega=0^{\circ}$ in the $[1 \overline{1} 0]$ direction). From the diffuse scattering spectra in all azimuths, we find the existence of nonspecular satellite peaks. These satellite peaks are located nearly symmetrically at both sides of the specular beam $\left(q_{\|}=0 \AA^{-1}\right)$ and can be clearly resolved. Since no satellite peaks are found in GaAs reference samples, these peaks can be attributed to diffuse scattering from the InAs QDs. We present GISAXS profiles in Fig. 2 for $\mathbf{q}_{\|}$parallel to [110], [1 10$]$, and [100] as an example. Figure 2 clearly shows high-order

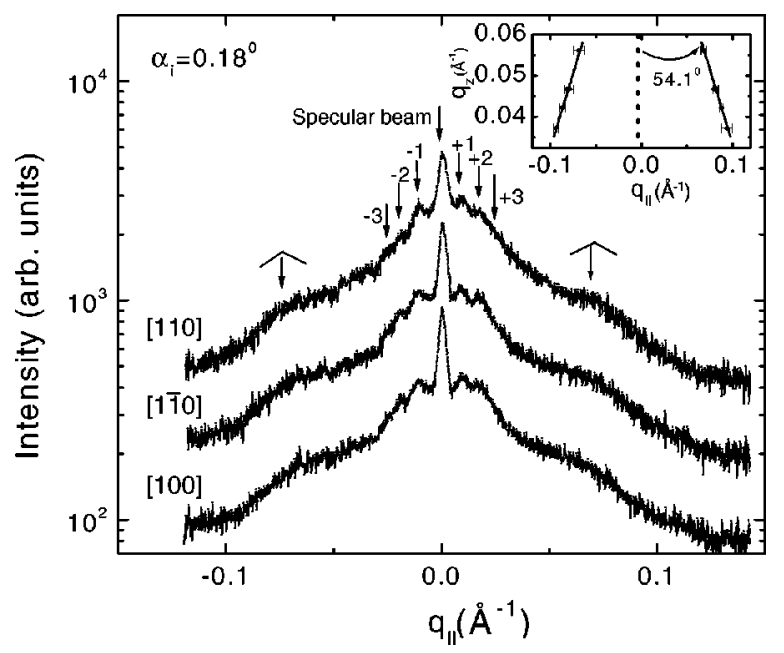

FIG. 2. Logarithmic GISAXS intensities along different $\mathbf{q}_{\|}$directions given at each profile. (At incidence angle $\alpha_{i}=0.18^{\circ}$ and exit angle $\alpha_{f}=0.40^{\circ}$.) The central peak at $q_{\|}=0$ is generated by the specular beam. The satellite peaks with higher orders are indicated by $\pm 1, \pm 2$, and \pm 3 . Additional broad facet spots at $q_{\|}= \pm 0.07 \AA^{-1}$ are indicated by $\wedge$ signs. The inset depicts the position of these facet spots in the $q_{\|}-q_{z}$ space (recorded at fixed

$\begin{aligned} & \alpha_{i} \text { ). } \\ & \text { Downloaded } 22 \text { Feb } 2008 \text { to 131.169.95.138. Redistribution subject to AlP license or copyright; see http://apl.aip.org/apl/copyright.jsp }\end{aligned}$

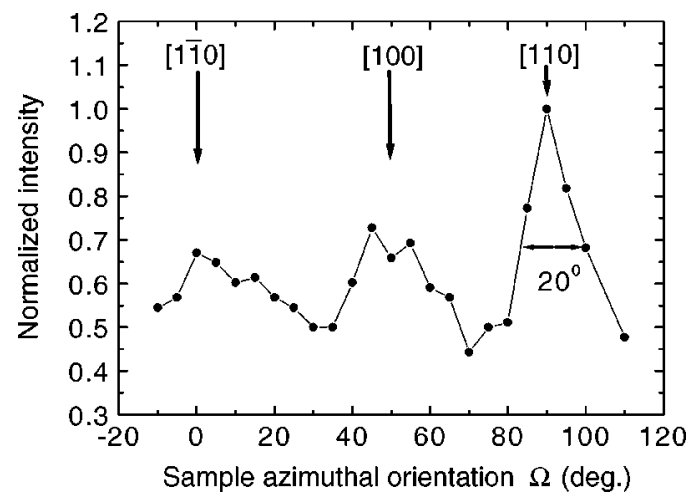

FIG. 3. Integrated satellite peak intensity vs sample azimuthal orientation $\Omega$. Satellite intensities have been normalized by the corresponding specular beam intensity.

satellite peaks in these three azimuths, i.e., $\pm 1, \pm 2$, and \pm 3 orders, indicating that the dot arrangement is well ordered.

The mean-dot-dot distance $\bar{d}$ is determined by the first satellite peak position with respect to the origin, i.e., $\bar{d}$ $=2 \pi / q_{\|}$. In the [1 $\left.\overline{1} 0\right]$ and [110] directions we find the same value $\bar{d}=64 \pm 2 \mathrm{~nm}$, whereas in the [100] direction the data yield $\bar{d}=72 \pm 2 \mathrm{~nm}$. We thus determine the ratio $R$ $=\bar{d}_{100} / \bar{d}_{1} \overline{1}_{0} \sim 1.13$. Intrinsically, a value of $R=1.0$ is expected for an isotropic dot distribution. Hence, $R=1.13$ reflects the presence of anisotropy in the ordering of the QDs. The anisotropy is smaller than in a perfect square-like lattice for which $R=1.41$ is expected. gorithm, we can determine the full width at half maximum (FWHM) values of the first-order satellite peaks. From AFM measurements at different surface spots, there is no evidence for large-scale fluctuations of the QD density. We thus regard the finite width of the satellite peaks to originate from local disorder among the nearest neighbors of each dot row in the chosen azimuth. With this assumption, correlation lengths of the dot distribution ${ }^{11}$ can then be obtained, which quantitatively reflect the degree of ordering in the lateral dot distribution. We find that the standard deviation of the dotdot distance ${ }^{12}$ in the [1 $\left.1 \overline{1} 0\right]$ and [110] directions is equal to $30 \%$, whereas along [100] it is $40 \%$. Assuming a shortrange-order mode of the correlation function, ${ }^{11}$ we then calculate the correlation length of $L=102 \pm 5 \mathrm{~nm}$ along the [1 $1 \overline{1} 0$ ] and [110] directions and $L=90 \pm 5 \mathrm{~nm}$ along [100], respectively. In combination with a larger mean-dot-dot distance along the [100] direction, this suggests a much weaker ordering along [100] than in the [1 $\overline{1} 0]$ and [110] directions.

The integrated intensities of the satellite peaks as a function of the azimuthal orientation are presented in Fig. 3. The intensity values are normalized to the corresponding specular beam intensities and have three maxima located at angles of $0^{\circ}, 45^{\circ}$, and $90^{\circ}$, respectively. These maxima reflect enhanced azimuthal ordering of the lateral dot distribution in the $[1 \overline{1} 0],[100]$, and [110] directions. We note that there is a higher intensity and a more narrow azimuthal peak width (about $20^{\circ}$ ) in the [110] direction as compared to [1 $\left.1 \overline{1} 0\right]$ and $[100]$ in Fig. 3. Although for both the [110] and [1 10$]$ directions we find the same mean-dot-dot distance and correlation length, this difference clearly indicates a better azimuthal ordering of the dot arrangement along [110] than o AIP license or copyright; see http://apl.aip.org/apl/copyright.jsp
Using an appropriate fit function and deconvolution al- 
along the $[1 \overline{1} 0]$ direction. We note that this observation is in agreement with our AFM Fourier analysis. The anisotropic lateral ordering of QDs may be caused by an anisotropic strain field around the QDs, in correspondence to previous studies on islands in InAs films below the critical thickness. $^{13}$

In addition to the satellite peaks we find broad maxima in the GISAXS spectra, located at $q_{\|}= \pm 0.07 \AA^{-1}$ and marked by $\wedge$ signs in Fig. 2 . We have investigated the dependence of the $q_{\|}$-peak position on $q_{z}$ for $\mathbf{q}_{\|}$parallel to [110], [110], and [100], respectively. The inset of Fig. 2 shows an example for the [110] direction. The peak positions can be described by a linear relation in the $q_{\|}-q_{z}$ space. This suggests that these broad intensity maxima are associated with crystal truncation rods (CTR) of the QD facets. The inclination angle of 54.1 $1^{\circ}$ with respect to the [001] surface normal strongly confirms that the peaks observed originate from $\{111\}$ QD facets. The same facet family is also observed for $\mathbf{q}_{\|}$parallel to [1 10$]$, whereas in the [100] direction $\{101\}$ facets are found. The observed facet spots originate from higher-order Bragg points and do not merge at (000) but at a finite $q_{z}$ value. This points to a partial strain relaxation within the InAs dots.

From the present experimental results, we propose that the InAs QDs are truncated pyramids with an octagonal basis and facet families of $\{111\}$ and $\{110\}$, in agreement with finite-element calculations ${ }^{10}$ and transmission electron microscopy studies by Grundmann et al. ${ }^{8}$ and by Chu et al. ${ }^{14} \mathrm{In}$ the literature, a number of different experimental techniques has been employed to gain information on the dot geometry, among which the most important are TEM, ${ }^{8,14}$ AFM, ${ }^{15-17}$ scanning tunneling microscopy, ${ }^{18,19}$ and RHEED. ${ }^{20,21}$ Yet, at present no consistent picture can be composed from the results reported. This may be originated by the fact that the contrast resolved by the different techniques originates from different physical properties. Moreover, even authors using the same technique propose different facets, which may indicate that the dots investigated are under different growth conditions like, e.g., different growth temperature, InAs deposit thickness, and different interdiffusion of In and $\mathrm{Ga}$ in the dots. In addition, from the FWHM of the facet CTR peaks an estimation can be given for the average facet size. ${ }^{11,22}$ In our case, we calculate a facet width of approximately $8 \pm 2 \mathrm{~nm}$, and from this, a dot height of about $5 \mathrm{~nm}$, which is consistent with our AFM data.

In summary, we present GISAXS experiments that reflect lateral ordering in a single layer of self-assembled InAs QDs on $\mathrm{GaAs}(001)$. Furthermore, in our experiments facet
CTR peaks are observed from which the dot facet shape can be inferred. Enhanced lateral ordering of the InAs QD distribution is found along [1 10$],[110]$, and [100]. We derive a mean-dot separation of $64 \mathrm{~nm}$ in $\langle 110\rangle$-like directions. The azimuthal dependence of the GISAXS intensity points to an anisotropic dot distribution with a large dot separation and a small correlation length in $\langle 100\rangle$-like directions. Moreover, the azimuthal dot distribution function is sharper in [110] than in other directions. This may indicate the presence of an anisotropic strain field around the QDs. The shape of InAs QDs is determined as a truncated octagonal-based pyramid.

The authors gratefully acknowledge financial support by the Graduiertenkolleg "Physik nanostrukturierter Festkörper,' and Dr. J. Wollschläger for helpful discussions.

${ }^{1}$ D. Heitmann and J. P. Kotthaus, Phys. Today 46, 56 (1993).

${ }^{2}$ D. Leonard, M. Krishnamurthy, C. M. Reaves, S. P. Denbaars, and P. M. Petroff, Appl. Phys. Lett. 63, 3203 (1993).

${ }^{3}$ A. A. Darhuber, V. Holy, J. Stangl, G. Bauer, A. Krost, F. Heinrichsdorff, M. Grundmann, D. Bimberg, V. M. Ustinov, P. S. Kopev, A. O. Kosogov, and P. Werner, Appl. Phys. Lett. 70, 955 (1997).

${ }^{4}$ Q. Xie, A. Madhukar, P. Chen, and N. P. Kobayashi, Phys. Rev. Lett. 75, 2542 (1995)

${ }^{5}$ B. J. Spencer and J. Tersoff, Phys. Rev. Lett. 79, 4858 (1997).

${ }^{6}$ V. Bressler-Hill, S. Varma, A. Lorke, B. Z. Nosho, P. M. Petroff, and W. H. Weinberg, Phys. Rev. Lett. 74, 3209 (1995).

${ }^{7}$ J. Tersoff, C. Teichert, and M. G. Lagally, Phys. Rev. Lett. 76, 1675 (1996).

${ }^{8}$ M. Grundmann, N. N. Ledentsov, R. Heitz, L. Eckey, J. Christen, J. Böhrer, D. Bimberg, S. S. Ruvimov, P. Werner, U. Richter, J. Heydenreich, V. M. Ustinov, A. Yu. Egorov, A. E. Zhukov, P. S. Kop'ev, and Z. I. Alferov, Phys. Status Solidi B 188, 249 (1995).

${ }^{9}$ J.-Y. Marzin and G. Bastard, Solid State Commun. 92, 437 (1994).

${ }^{10}$ N. Moll, M. Scheffler, and E. Pehlke, Phys. Rev. B 58, 4566 (1998).

${ }^{11}$ M. Schmidbauer, Th. Wiebach, H. Raidt, M. Hanke, R. Köhler, and H. Wawra, Phys. Rev. B 58, 10523 (1998).

${ }^{12}$ J. Wollschläger, Surf. Sci. 328, 325 (1995).

${ }^{13}$ V. Bressler-Hill, A. Lorke, S. Varma, P. M. Petroff, K. Pond, and W. H. Weinberg, Phys. Rev. B 50, 8479 (1994).

${ }^{14}$ L. Chu, M. Arzberger, G. Böhm, and G. Abstreiter, J. Appl. Phys. 85, 2355 (1999).

${ }^{15}$ D. Leonard, K. Pond, and P. M. Petroff, Phys. Rev. B 50, 11687 (1994).

${ }^{16}$ N. P. Kobayashi, T. R. Ramachandran, P. Chen, and A. Madhukar, Appl. Phys. Lett. 68, 3299 (1996).

${ }^{17}$ J. M. Moison, F. Houzay, F. Barthe, L. Leprince, E. Andre, and O. Vatel, Appl. Phys. Lett. 64, 196 (1994).

${ }^{18}$ Qi-k. Xue, K. Hasegawa, H. Kiyama, and T. Sakurai, Jpn. J. Appl. Phys., Part 1 38, 500 (1999).

${ }^{19}$ P. B. Joyce, T. J. Krzyzewski, G. R. Bell, B. A. Joyce, and T. S. Jones, Phys. Rev. B 58, R15981 (1998).

${ }^{20}$ Y. Nabetani, T. Ishikawa, S. Noda, and A. Sasaki, J. Appl. Phys. 76, 347 (1994).

${ }^{21}$ H. Lee, R. Lowe-Webb, W. Yang, and P. C. Sercel, Appl. Phys. Lett. 72, 812 (1998).

${ }^{22}$ S. Song and S. G. J. Mochrie, Phys. Rev. Lett. 73, 995 (1994). 